\title{
Study of Noise Mitigation for Underwater Acoustic Channel
}

\author{
Huakui Wang ${ }^{1, \text { a }}$, Xiaoyan Zhang ${ }^{2, b}$, Yanhua Liu' ${ }^{2, c}$, Haixin sun ${ }^{2}$ \\ ${ }^{1}$ Sci. \& Tech. on Underwater Acoustic Antaganizing Laboratory, Zhanjiang, China \\ ${ }^{2}$ College of information science and technology, Xiamen university, Xiamen, China

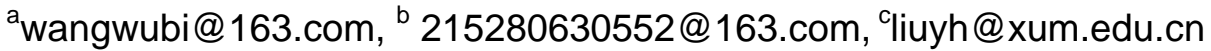

Keywords: compressed sensing; impulse noise; single-frequency noise; underwater acoustic channel

\begin{abstract}
Impulse noise and large scale single-frequency noise have an enormous effect on underwater acoustic communication and they often exist in the received signal. This paper focuses on noise mitigation when the two kinds of noise both exist. These interferences are sparse, and they have lager amplitudes, which are able to be weakened by using compressed sensing theory reasonably. Based on orthogonal matching pursuit (OMP), this paper uses the observed value to reconstitute the noise and suppress them. Experimental results performed with numerical simulation and pool-trial data are provided. The results show the improvement of the proposed algorithm under impulse noise mitigation and large scale single-frequency noise mitigation can get more performance gain margin. Through the estimation and suppression of them, the robustness is improved.
\end{abstract}

\section{Introduction}

Noise can be divided into internal and external disturbances [1].Single carrier transmission of intersymbol interference and multicarrier transmission intercarrier interference are internal interference, the influence of external interference is from the environment. Disturbances coming from external sonar and Marine environment influence underwater acoustic communication [2] [3], seriously affecting the performance of the system. Therefore, it has great significance to eliminating interference for underwater acoustic communication. This paper mainly focuses on eliminating the influence of external disturbance on the underwater acoustic communication system.

And noise elimination has been extensively studied in the wireless communication. Clipping method was the mainly tradition impulse noise suppression method. An application of Reed-Solomn(RS) coding scheme for impulsive noise mitigation was proposed by using the time-domain clipping in [4].In [5], performing joint erasure marking and Viterbi decoding, the proposed scheme was improved in [4], but at the cost of high computational complexity. Iterative strategy was proposed in [6]; On this basis, the decoder adopted a syndrome in order to increase the convergence speed was proposed in [7]. However, these two methods are assumed in the case of the ideal channel. Article [8] proposed a pre-algebraic coding and frequency interpolation techniques, that is, the use of zero-frequency point to detect and estimate the impulse noise, the disadvantage is that the zero frequency is very sensitive to background noise. Article [9] used the compressed sensing technology, using loophole in OFDM modulation signal carrier for impulse noise estimates. Article [3] that the impulse noise is a sparse vector, use compressed samples to reconstruct, this method has a more flexible system design and more robust. However, previous studies mainly consider the case of a presence of noise, but in the underwater acoustic channel, large single frequency noise and impulse noise interference can exist at the same time, some of the major factors that affect the communication performance. So the underwater acoustic communication, while eliminating a substantial single-frequency noise and impulse noise to improve the robustness of underwater acoustic communication system is of great significance.

In this paper, the use of compressed sensing theory eliminate the effects of impulse noise and substantial single-frequency noise in OFDM system. In impulse noise and single by orthogonal matching pursuit (OMP) algorithm frequency noise reconstructed using observations on the 
receiving end to eliminate noise, impulse noise and eliminate a significant impact on the single-frequency noise signals, improve system performance.

\section{Compressed sensing theory}

Based on compressive sensing theory, without losing information required to reconstruct the original signal, sparse signal can use traditional sampling observation of low dimension vector $y \in R^{M}$ instead of discrete sequences $x \in R^{N}$, M for the observation points, $N$ as the signal points, and $M<N$.

The essence of the compressed sampling is based on the observation of a matrix $\Phi$ to get observation vectors $\mathrm{y} \in \mathrm{R}^{\mathrm{M}}$.

$$
y=\Phi x=\Phi \psi s=\Theta s
$$

Where $\Theta=\Phi \psi$, is a dictionary of size $M \times N$. If $\Theta$ is sufficiently incoherent ,the information of $x$ will be embedded in $y$ such that it can be perfectly recovered with high probability[10]. $x=\Psi_{s}, \mathrm{~s}$ is the coefficient of $x$ on the orthogonal basis $\psi$.

\section{System model}

In OFDM system, when the additive white Gaussian noise and external interference exist, mainly affect the system performance of external noise, thus eliminating external noise to improve the system performance has an important role. Therefore, the discrete-time channel model can be expressed as

$$
y_{k}=\sum_{l=0}^{L} h_{l} x_{k-l}+z_{k}+e_{k}
$$

Where $\left\{x_{k}\right\}$ are the transmitted signals and $\left\{y_{k}\right\}$ are the received signal sequence, $\left\{z_{k}\right\}$ for the additive white Gaussian noise sequence, $\left\{e_{k}\right\}$ to external noise sequence, that are impulse noise and substantial single-frequency noise.

\section{Noise reconstruction}

OMP algorithm is a matching pursuit (MP) algorithm to improve the algorithm to improve the computing speed, and easy to implement.

OMP algorithm is described in detail follows [11]:

Step 1: Initialization, define $r$ is the residual vector, $P$ is position vector, so $r_{0}=y, P_{0}=\varnothing$, where $\varnothing$ stands for null set.

Step 2: for each $i(i=1,2, \cdots K)$, OMP chooses another index $\lambda_{i}$ by solving an easy optimization problem.

$$
\lambda_{\mathrm{i}}=\arg \max \left|\Theta^{T} r_{i-1}\right|
$$

Step 3: on update stage, update $A_{i}$, according to $A_{i}$ and $\Theta_{l}\left(l=P_{i}\right)$.

$$
A_{i}=\left[A_{i-1}, \Theta_{l}\right]
$$

For each column $l$, find $s_{i}$,

$$
s_{i}=\underset{s}{\arg \min }\left\|y-\Theta_{i} s\right\|
$$

Step 4: the residual $r_{i}$ at each step can be expressed as 


$$
r_{i}=y-\Theta_{i} s_{i}
$$

Step 5: if $i<K, \quad i=i+1$ and return to step 2 cycle is repeated; if $i=K$ and stop iteration.

According to the above steps, noise interference can be estimated easily. Based on OMP, impulse noise is estimated by using the null-subcarrier and the large scale signal frequency noise is estimated directly without domain transformation because of the sparse in the frequency domain.

Two separate optimization methods are proposed in this paper. One is that we estimate impulse noise firstly, and then the estimation of large scale single frequency noise can be obtained by using the measurements on the subcarriers (shown in fig.1). The other is that large scale single frequency noise is estimated firstly and then the impulse noise estimation is also done as before (shown in fig.2).

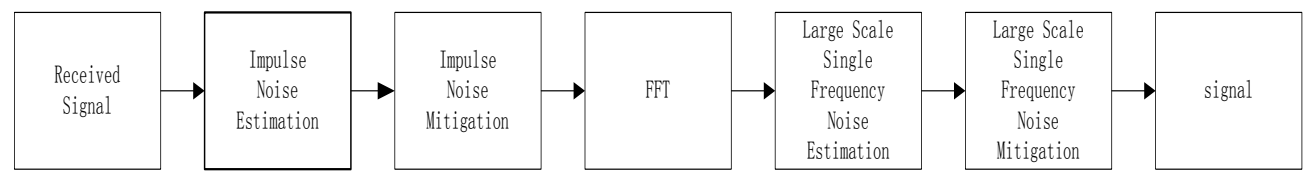

Fig.1 Impulse noise mitigation firstly at the receiver

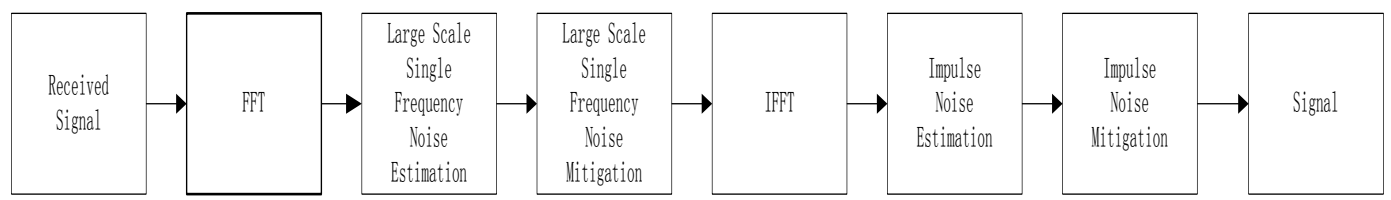

Fig.2 Large scale single frequency noise mitigation firstly at the receiver

\section{Simulation}

In order to verify the performance of the proposed method, we take the simulation experiments, including the multipath channel consists of five discrete path, where the inter-arrival time follows an exponential distribution with a mean of 1ms. Multi-path channel gain Rayleigh distribution, its average power decays exponentially with time delay. The CP-OFDM signal parameters are follows: Out of 1024 subcarriers, 96 are null subcarriers with 24 on each edge for band protection and 48 distributed evenly in the middle. The impulse noise and large scale single frequency noise are both generated with 5 impulses imposed with random positions on the K=1024 OFDM baseband samples.

The data symbols are drawn from a QPSK constellation, and 256 pilot data are used for channel estimation. A 64-state rate $-1 / 2$ convolutional code is used for channel coding [12]. The bit-error-rate(BER) after Viterbi decoding will be used as the performance metric.

In the simulation, firstly the receive received the signal denoised, and then reuse the signal denoised to estimate and balance based on the compressed sensing channel. Fig.3 shows that BER comparison of noise mitigation as a function of SNR. Black line represents the time domain impulse noise signal contained in a single frequency noise or substantial interference, the receiver does not eliminate the noise interference simulation (method a); The blue line represents estimate impulse noise firstly, and then the estimation of large scale single frequency noise ( method b); The red line represents large scale single frequency noise is estimated firstly and then the impulse noise estimation is also done as before (method c). Figure 3 shows that our algorithm can get great performance gain from the beginning of $2 \mathrm{~dB}$, the performance of this algorithm can improve the bit error rate is about 10 times as $16 \mathrm{~dB}$. And with separate large scale single frequency noise mitigation firstly, the proposed received has a better performance than the another method. Figure 4 shows that BER comparison of noise mitigation as a function of SIR. And we can see the system performance gain with the SIR increases gradually increases. In the signal interference ratio is $-4 \mathrm{~dB}$, the noise cancellation performance of the bit error rate of about $16 \%$. 
The signal-to-noise ratio (SNR) and signal-to-impulse noise ratio( SIR) are defined as:

$$
\begin{aligned}
& S N R=\frac{E\left[\|x\|^{2}\right]}{E\left[\|z\|^{2}\right]} \\
& \text { SIR }=\frac{E\left[\|x\|^{2}\right]}{E\left[\|e\|^{2}\right]}
\end{aligned}
$$

Where $x$ is the signal sequence, $z$ is the additive Gaussian white noise sequence and $e$ is the external noise sequence.

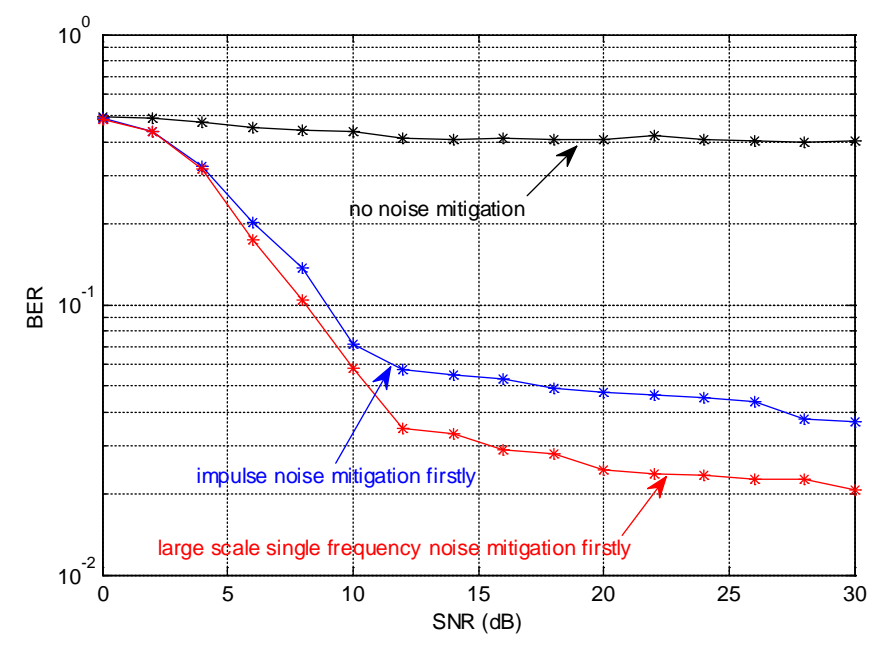

Fig.3 BER comparison of noise mitigation as a function of SNR

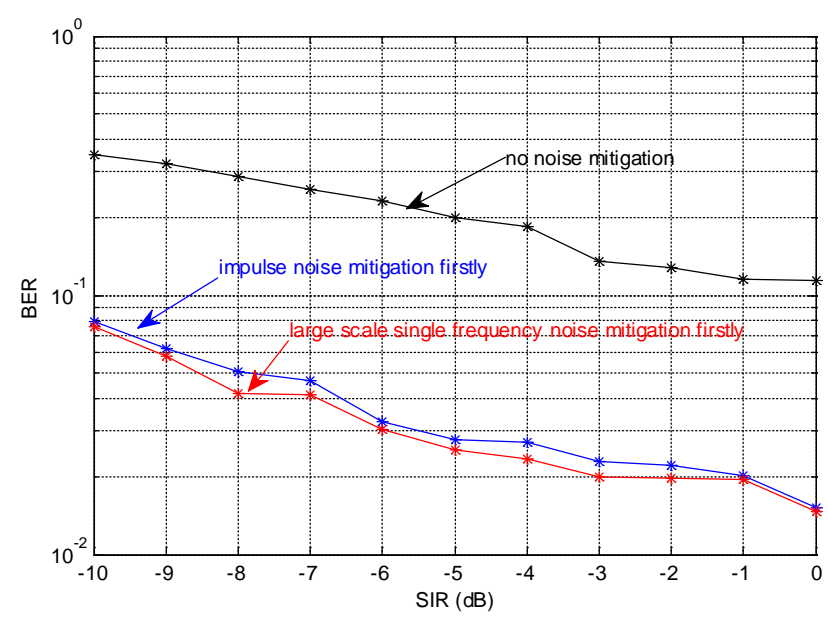

Fig.4 BER comparison of noise mitigation as a function of SIR

\section{Pool experimental results}

There are one transmitter and one receiving hydrophone and both located at the depth of $0.8 \mathrm{~m}$ below the surface and the distance between them is $7 \mathrm{~m}$. Transmitting and receiving transducer bandwidth is $6 \mathrm{kHz}$. Transmit carrier frequency signal is $11 \mathrm{kHz}$, and through quadrature phase shift keying (QPSK) modulation modulated signal, the receiver sampling rate of $66 \mathrm{kHz}$. The received signal using channel estimation and equalization based on compressive sensing theory.

The poor experiment investigates the performance of our proposed methods. Comparing the pool experimental results in TABLE I, it is observed that the proposed methods have a better performance on noise mitigation and with separate large scale single frequency noise mitigation firstly, the proposed received has a better performance. 
Table I Pool Experimental Results

\begin{tabular}{|c|c|c|}
\hline Method a & Method b & Method c \\
\hline 0.4279 & 0.2539 & 0.1788 \\
\hline 0.3432 & 0.2197 & 0.1952 \\
\hline 0.3710 & 0.1618 & 0.1593 \\
\hline 0.2948 & 0.2775 & 0.2766 \\
\hline 0.3018 & 0.0765 & 0.0706 \\
\hline
\end{tabular}

\section{Summary}

This paper applied compression perception theory which is based on the OMP algorithms, studying the signal of the external noise elimination. Simulation and pool experimental results show that the method put forward in this paper to eliminate impulse noise significantly can improve the robustness of underwater acoustic communication system.

\section{References}

[1] H. Sun, W. Shen, Z. Wang, S. Zhou, X. Xu, and Y. Chen, Joint carrier frequency offset and impulse noise estimation for underwater acoustic OFDM with null subcarriers[J]. Oceans, 2012, Virginia, US

[2] J. Catipovic, M. Johnson, and D. Adams, Noise cancelling performance of an adaptive receiver for underwater communications[C]. In Proceedings of the Symposium on Autonomous Underwater Vehicle Technology, July 1994, pp. $171-178$.

[3] M. Chitre, S. H. Ong, and J. Potter, Performance of coded OFDM in very shallow water channels and snapping shrimp noise[C]. In Proceedings of MTS/IEEE OCEANS, vol. 2, 2005, pp. 996-1001

[4] J. A. T. Zogakis, P. Chow and J. Cioffi, Impulse noise mitigation strategies for multicarrier modulations[C]. In Proc. of Intl. Conf. on Commun., May. 1993, p. 784C788.

[5] W. H. M. T. Li and M. H. Siu, A joint approach to erasur e marking and viterbi decoding for impulsive noise channels[J]. In IEEE Workshop on Signal Processing Advances in Wireless Communications, Jun. 2003

[6] S. Zhidkov, Impulsive noise suppression in OFDM-based communication systems[J]. IEEE Trans. Consum. Electron., vol. 49, no. 4, pp.944-948, Nov. 2003

[7] A. Mengi and A. Vinck, Successive impulsive noise suppression in OFDM [C]. In Proc. of IEEE Intl. Symp. on Power Line Communications and Its Applications, Mar. 2010, p. 3337

[8] M. Ester, H.-P. Kriegel, J. Sander, and X. Xu, A density-based algorithm for discovering clusters in large spatial databases with noise [C]. In Proc. 2nd Int. Conf. on Knowledge Discovery and Data Mining, 1996, pp. 226-231

[9] G. Caire, T. Y. Al-Naffouri, and A. Narayanan, Impulse noise cancellation in OFDM: An application of compressed sensing [C]. In Proceedings of Intl. Symposium on Information Theory, July 2008, pp. 1293-1297

[10] Baraniuk R G. Compressive Sensing [J]. IEEE Signal Processing Magazine, 2007, 24 (4): 118121

[11] Yang D P, Li H S, Peterson G D, et al. Compressed Sensing Based UWB Received : Hardware Compressing of a Serial Circuit Power Divide [J]. Radar Science and Technology, 2009,7(5): 389-391.(in Chinese)

[12] Zhu X W. Statistical properties of multi-path channel convolution code [J]. Radar Science and Technology,1992, 17(4): 278-284. (in Chinese) 